\title{
Growth Module in the Pediatric Preclerkship Educational Exercises (PRECEDE) Curriculum
}

David W. Cooke, MD*, Eric Balighian, MD, Stacy Cooper, MD, Michael Barone, MD, MPH, Robert Dudas, MD, Emily Frosch, MD, Justin Jeffers, MD, Rosalyn Stewart, MD, W. Christopher Golden, MD

*Corresponding author: dcooke@jhmi.edu

\section{Abstract}

Introduction: The Johns Hopkins Pediatrics Clerkship developed this growth module as part of the PRECEDE (preclerkship educational exercises) curriculum, with the primary goal of providing students with experiential, explicit, and standardized instruction in essential pediatric clinical skills to better prepare them to utilize these skills during their clerkship. Methods: This 2-hour growth module begins with a 45minute didactic overview of growth, including discussion of normal growth, normal variants of growth, and disorders that affect growth, and contains interactive elements to engage the students. Students then divide into groups of four to six, each with a faculty facilitator to work through three cases in a guided discussion to explore specific aspects of growth that may be encountered in a pediatric evaluation.

Results: In a survey of 238 students, $97-100 \%$ agreed or strongly agreed with eight positive assessments of the module. Likewise, $79 \%$ of students rated the module as excellent, the remainder rating the module as good. Discussion: From the perspectives of students and instructors, the implementation of this new module was very successful in its delivery of educational content. These cases were designed to give the students experience in plotting growth parameters against normative data and to gain familiarity with pediatric growth curves in order to identify growth abnormalities. The cases also aimed to reinforce the importance of using growth data in pediatric patients in order to both generate a differential diagnosis for a growth disorder and to modify a differential diagnosis generated by a chief complaint based on growth data.

\section{Keywords}

Growth, Case-Based Learning, Pediatrics

\section{Educational Objectives}

By the end of this session, pediatric clerkship students will be able to:

1. Accurately plot growth data against normal standards.

2. List three factors that contribute to normal variants of growth in children.

3. Generate an initial differential diagnosis based on a set of growth curves for a child.

4. Compare and contrast how growth curves influence the differential diagnosis for a given chief complaint.

\section{Introduction}

The Johns Hopkins University School of Medicine's Pediatrics Clerkship developed the PRECEDE (preclerkship educational exercises) curriculum with the primary goal of providing students with experiential, explicit, and standardized instruction in essential pediatric clinical skills to better prepare them to incorporate these skills into their clerkship experiences. This growth workshop is one of a series of modules offered during PRECEDE. As the name implies, all students assigned to the pediatric clerkship for a given academic session participate in these PRECEDE modules immediately prior to joining their individual assignments, whether that be an inpatient setting or an outpatient setting. In the Johns Hopkins curriculum, the growth module is the very first module in the pediatrics PRECEDE curriculum.

Cooper S, et al. Growth module in the pediatric preclerkship educational exercises (PRECEDE) curriculum. MedEdPORTAL. 2018;14:10687. https://doi.org/10.15766/mep_23748265.10687

Copyright: $\odot 2018$ Cooke et al. This is an open-access publication distributed under the terms of the Creative Commons Attribution-NonCommercialShare Alike license.

Appendices

A. PRECEDE Growth.pptx

B. PRECEDE Growth Lecture Guide.docx

C. PRECEDE Growth Student Handout.doc

D. PRECEDE Growth Cases Student Packet.doc

E. PRECEDE Growth Cases Instructor Packet.doc

F. PRECEDE Growth Student Version.pptx

All appendices are peer reviewed as integral parts of the Original Publication. 
The development of the PRECEDE curriculum began with a needs assessment which asked an initial cohort of students "What clinical skills or knowledge specific to pediatrics do you wish you had been explicitly taught, with opportunity for practice and feedback, before you started to see patients on the pediatric clerkship?" This local needs assessment was further refined by discussion with clerkship directors from other disciplines at our institution to avoid redundancies in the delivery of clinical skills instruction. ${ }^{1}$

Because of the value in evaluating growth in pediatric patients, the American Academy of Pediatrics recommends monitoring growth by measuring height/length and weight (and head circumference in children under 2 years of age) at all health supervision visits. ${ }^{2}$ Students at Johns Hopkins have preclinical lectures related to normal and abnormal growth. However, the curriculum designers felt that augmenting the students' preclinical instruction with both didactic and case-based investigations of growth in children just before starting their clinical pediatric experience would be useful. Given the importance of evaluating growth in virtually all pediatric encounters, the educational team aimed to have the students start the clerkship prepared with this skill. In addition, utilizing growth assessment in clinical encounters is unique to pediatric medicine (aside from monitoring fetal growth in obstetrics), and therefore serves as a very appropriate introduction to the pediatric clerkship. Further, by having this session just before embarking on their clerkship, this session not only prepares students to think about growth as an important aspect of pediatrics, but it also allows them to quickly reinforce this concept and the learned skill set by implementing anthropometrics immediately into their clinical practice.

This workshop was designed to incorporate the Council on Medical Student Education in Pediatrics' curricular objectives on growth, ${ }^{3}$ which state that all students should be able to:

1. Describe variants of normal growth in healthy children (e.g., familial short stature and constitutional delay).

2. Identify and describe abnormal growth patterns based on the family growth history and the child's previous growth (e.g., microcephaly, macrocephaly, short stature, obesity, growth abnormalities related to specific physical findings).

3. Identify failure to thrive and overweight/obesity in a child or adolescent using body mass index and other growth measures, and outline the differential diagnosis and initial evaluation.

4. Demonstrate ability to measure and assess growth including height/length, weight, head circumference, body mass index in patient encounters using standard growth charts.

An overarching concept of this workshop is an emphasis on the importance of assessing growth parameters at every pediatric encounter, whether it be a well child visit or an acute care encounter, and then to use that data as another factor in the overall assessment of the child's health status.

Given the importance of assessing children's growth as a marker of health, it is not surprising that there are other MedEdPORTAL resources related to teaching growth evaluation skills to medical students. ${ }^{4-7}$ Hanson et al. address growth in their curriculum but focus overall on the role of the health supervision visit, placing a greater emphasis on developmental assessments. ${ }^{4}$ In another resource, Warrier and colleagues look at nutritional aspects of growth, ${ }^{5}$ whereas our curriculum evaluates the potential impact of both nutritional and non-nutritional factors on growth, and also prepares students to evaluate ponderal and linear growth.

The two resources by MacPherson et al. are most similar to our PRECEDE module. ${ }^{6,7}$ They present didactic material in a podcast format reinforced with specific cases, such as a case of failure to thrive, ${ }^{6}$ and a case of delayed puberty. ${ }^{7}$ In contrast, our module has the advantages of providing the visual material in the format of a PowerPoint presentation, and includes instructor-guided small-group discussions that highlight general principles for utilizing growth assessment during pediatric encounters. This module also 
complements our other PRECEDE modules in highlighting the variability that can be seen in pediatric encounters. $^{8}$

\section{Methods}

This workshop was designed to be presented in a large room, where the entire group could view a PowerPoint presentation and subsequently break out into small groups of four to six students with each group working independently with a separate faculty facilitator. These small groups could be gathered around a table, but could also work by gathering chairs in a circle. The duration of the workshop was approximately 2 hours, allowing for a break between the didactic and small-group sections.

The module is composed of six resource files. Appendices $A$ and $F$ are the two PowerPoint presentations for the didactic presentation. Appendix A may be used if the didactic session is presented to the students by a lecturer. Appendix F may be used in a flipped-classroom approach. The module also contains four document files to be printed. Prior to the session, the Faculty Lecture Guide (Appendix B) is printed for the didactic presenter. One copy per student of the Student Handout (Appendix C) and the Growth Cases Student Packet (Appendix D) are printed. One copy per small group instructor of the Growth Cases Instructor Packet is also printed (Appendix E).

The workshop began with one faculty member giving a didactic presentation using the PowerPoint slides and the provided presenter guide. While most of this presentation was in lecture format, there were sections at the end that involved active participation by the students. The first opportunity was regarding laboratory testing in the evaluation of a child with concerns of poor growth. The students were asked to use the information already presented, as well as their prior knowledge from preclinical learning (or from other clinical rotations they may have completed) to suggest what diagnoses would be tested for by the typical laboratory tests obtained in the evaluation of poor growth. This exercise challenged the students to begin thinking about both the broad differential diagnosis for poor growth, and the range of information provided by each laboratory test. The didactic presentation ended by having the students plot growth data using the growth curves in the student handout and begin to think about using the growth curves in evaluating patients. This exercise also demonstrated how to incorporate the child's parents' heights into the assessment.

After the didactic presentation (and a short break), the students gathered in groups of four to six with a faculty facilitator. The students were given the Growth Cases Student Packet (Appendix D), while the faculty worked from the Growth Cases Instructor Packet (Appendix E).

\section{Case 1}

The first case in the small-group session had the students review the sets of growth curves (head circumference, length, and weight) of three infants. In case $1 \mathrm{~A}$, everything followed the 50th percentile except head circumference, which was rising on the growth curve; in case $1 \mathrm{~B}$ everything followed the 50th percentile; and in case $1 \mathrm{C}$ everything followed the 50th percentile except weight, which was falling on the growth curve. The facilitator then guided the students through a discussion of the curves, including appreciating a differential diagnosis for pathologic processes while also considering normal, nonpathologic variants. This discussion began with considerations based just on the growth curves, then focused on how to use the growth curves to guide an assessment based on a chief complaint (e.g., "throws up frequently"). In addition to allowing students to integrate growth data into an assessment based on other clinical information, this case also provided an opportunity to share information with students about gastroesophageal reflux, something that will undoubtedly be revisited at other times during their pediatrics clerkship.

\section{Case 2}

The second case in the small group session had the students review the growth curves of an infant whose length and weight are both decreasing on the curves. The facilitator again guided the students through a 
differential diagnosis, with the intent of highlighting the more subtle nuances in growth disorders. In the curves for case $2 \mathrm{~A}$, the length was affected more than the weight; in $2 \mathrm{~B}$, the weight was affected more than the length. This case also brought parental size into the assessment of childhood growth, with the students practicing the mid-parental height calculation, generating a target height range, and using that information to assess a child's growth.

\section{Case 3}

The third and final case allowed students to appreciate the necessity of using growth curves to interpret anthropometric data. It focused on identifying childhood obesity, and through this exercise the students began to understand the difficulty in identifying obesity simply by sight or weight. Instead, they needed to calculate and plot the body mass index percentile to identify overweight and obese children. While every student could work through the data for all six patients presented in case 3 , in the interest of time, each student worked through the data on one or two of the patients. They used the growth curves they were provided in the Student Handout (Appendix C) provided prior to the didactic presentation at the start of the session.

\section{Results}

We evaluated the impact of the PRECEDE growth workshop by surveying students in the pediatric clerkship from 2010 to 2012. In total, 238 students completed the survey. Each question had four options the student could choose: strongly disagree, disagree, agree, and strongly agree. All students agreed that the session was educationally valuable (Table). Across the range of questions that were asked, the students responded with very positive assessment of this workshop. When rating the overall quality of the session, students had four choices: poor, fair, good, or excellent. Seventy-nine percent rated it as excellent, and $21 \%$ rated it as good.

Table. Results of Student Survey $(N=238)$

Item

\begin{tabular}{cc} 
Agree (\%) & Strongly Agree (\%) \\
\hline 23 & 76 \\
24 & 76 \\
22 & 78 \\
2 & 78 \\
15 & 82 \\
14 & 85 \\
17 & 82 \\
15 & 82
\end{tabular}

in session met the stated objectives.

This session increased my skills related to this topic.

Having had this session will improve my ability to function as a clinical clerk on the wards.

This session was educationally valuable.

The instructors provided a supportive learning environment.

Active student participation was encouraged.

The instructors enabled me to accomplish the learning objectives for the session.

The instructors' teaching methods stimulated my own critical thinking.

\section{Discussion}

This growth workshop is the very first session in our PRECEDE curriculum on the pediatrics clerkship at the Johns Hopkins University School of Medicine. While that timing is not critical to its utility, this workshop is a logical introduction to the pediatric clerkship, as it presents an aspect of pediatrics that is relevant to almost all pediatric encounters, and that clearly differentiates pediatrics from adult medicine. The workshop starts by giving students the knowledge needed to recognize abnormalities and normal variants of growth, and subsequently engages students in working through scenarios where they have to put that knowledge to use. These exercises are designed to specifically emphasize how information about a child's growth can impact the assessment of a child's presentation.

Evaluation by students who completed this session indicated that this module was successful in achieving the objectives and in preparing them for their clerkship experience. Ideally, we would have results from assessments that measure an improvement in the ability of a student to recognize a pathologic growth pattern from a healthy growth pattern (including the various normal variations) and to generate an initial differential diagnosis for a given presentation. Unfortunately, such higher-order outcomes are difficult to capture during the experiential portion of the clerkship. In the future, simulated cases could be used to more definitively document the workshop's effectiveness as an educational resource. 
One limitation of this workshop is that it begins with a predominantly lecture-like didactic session, which lacks the active-learning approach that more effectively engages learners. This didactic, however, does contain specific interactive components to engage the students and also ensures that all the students have the foundational knowledge needed to acquire value out of the small-group discussions that follow. If desired, an audience response system could be utilized for some of these audience queries. If a flipped classroom approach is used, rather than have a lecturer give the didactic presentation, the students can be provided with the student version of the PowerPoint presentation (Appendix F) and the Student Handout (Appendix C) to work on independently prior to the meeting for the small-group case sessions. This student version of the PowerPoint presentation is modified slightly from the lecture version to allow a student to view the presentation independent of a lecturer. While some of the finer points that can be provided by an experienced facilitator would be missed, the main objectives should be able to be met by the independent review, and may better meet the time and resource requirements of some programs.

Another limitation is the high number of instructors required. This is, of course, necessary to maintain the advantages of small-group discussion. While we have used faculty to fill these instructor roles, second- or third-year pediatric residents or appropriate pediatric subspecialty fellows could also be utilized as instructors.

The case discussions in the small groups are not fully formed cases with an ultimate diagnosis, but are presented with variations that are then compared. This approach encourages the students to think in broader terms about how differences in growth patterns might impact the differential diagnosis for a growth disorder, and how differences in other clinical information (such as the history) might result in a different interpretation of the growth pattern. The expectation is that the students then use these principles when evaluating the patients they see during their clerkship.

The cases in this workshop that evaluate growth patterns are of infants. While there might be value in developing an additional case utilizing an older child, this change would have to be weighed against the added time necessary to go through an additional case. Since a major emphasis of this workshop is on the utility of evaluating growth to identify disorders other than just primary growth disorders, a case that would fit well in this workshop would be an older child with abdominal complaints. Growth curves could be developed that would match what would be expected for a child with functional abdominal pain versus those of a child with inflammatory bowel disease versus those of a child with constipation from hypothyroidism. However, the principles that such a case would highlight are already demonstrated in cases 1 and 2 of this workshop. If desired, a case involving an older child could be used as a discussion point within the context of the existing small-group cases.

In summary, we have developed a workshop to evaluate growth in pediatric patients. Our experience, which is confirmed by student evaluations, is that this is an effective introduction to a pediatric clerkship and prepares students for pediatric clinical experiences.

David W. Cooke, MD: Associate Professor, Department of Pediatrics, Johns Hopkins University School of Medicine

Eric Balighian, MD: Assistant Professor, Department of Pediatrics, Johns Hopkins University School of Medicine

Stacy Cooper, MD: Assistant Professor, Departments of Oncology and Pediatrics, Johns Hopkins University School of Medicine

Michael Barone, MD, MPH: Associate Professor, Department of Pediatrics, Johns Hopkins University School of Medicine

Robert Dudas, MD: Associate Professor, Department of Pediatrics, Johns Hopkins University School of Medicine

Emily Frosch, MD: Associate Professor, Department of Psychiatry and Behavioral Sciences, Johns Hopkins University School of Medicine

Justin Jeffers, MD: Assistant Professor, Department of Pediatrics, Johns Hopkins University School of Medicine 
Rosalyn Stewart, MD: Associate Professor, Departments of Medicine and Pediatrics, Johns Hopkins University School of Medicine

W. Christopher Golden, MD: Assistant Professor, Department of Pediatrics, Johns Hopkins University School of Medicine

\section{Disclosures}

Dr. Golden reports personal fees from UBM/Advanstar Communications, personal fees from Wolters Kluwer, outside the submitted work.

Funding/Support

None to report

Ethical Approval

Reported as not applicable.

\section{References}

1. Dudas RA, Colbert-Getz JM, Balighian E, et al. Evaluation of a simulation-based pediatric clinical skills curriculum for medical students. Simul Healthc. 2014;9(1):21-32. https://doi.org/10.1097/SIH.0b013e3182a89154

2. Hagan JF, Shaw JS, Duncan PM, eds. Bright Futures: Guidelines for Health Supervision of Infants, Children, and Adolescents. 4th ed. Elk Grove, II: American Academy of Pediatrics; 2017.

3. Curriculum competencies and objectives. Council on Medical Student Education in Pediatrics Web site. https://www.comsep.org/educationalresources/currobjectives.cfm. Updated 2017.

4. Hanson J, Balog E, Pelzner M. Pediatric health supervision curriculum: instructor's guide, student resources, faculty resources and assessment tools. MedEdPORTAL. 2014;10:9752. https://doi.org/10.15766/mep_2374-8265.9752

5. Warrier K, Mychaliska K, Schiller J, Mcbee-Orzulak F, Frei N. Pediatric nutrition team-based learning module.MedEdPORTAL. 2010;6:8358. https://doi.org/10.15766/mep_2374-8265.8358

6. MacPherson P, Lewis M. PedsCases - a learning module for the evaluation of a child with failure to thrive for medical students. MedEdPORTAL. 2010;6:7912. https://doi.org/10.15766/mep_2374-8265.7912

7. MacPherson P, Marks S, Couch R. PedsCases - a learning module for evaluation of pediatric short stature and delayed puberty for medical students. MedEdPORTAL. 2010;6:7976. https://doi.org/10.15766/mep_2374-8265.7976

8. Balighian E, Barone M, Cooke D, et al. Interpretation of data workshop in the pediatric preclerkship educational exercises (PRECEDE) curriculum. MedEdPORTAL. 2016;12:10496. https://doi.org/10.15766/mep_2374-8265.10496

Received: September 28, 2017 | Accepted: January 24, 2018 | Published: February 27, 2018 